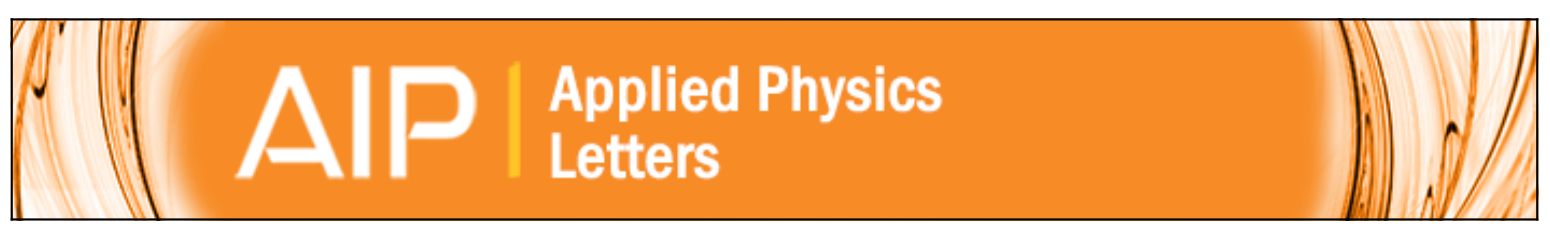

\title{
Multimodal system for harvesting magnetic and mechanical energy
}

Shuxiang Dong, Junyi Zhai, J. F. Li, D. Viehland, and S. Priya

Citation: Applied Physics Letters 93, 103511 (2008); doi: 10.1063/1.2982099

View online: http://dx.doi.org/10.1063/1.2982099

View Table of Contents: http://scitation.aip.org/content/aip/journal/apl/93/10?ver=pdfcov

Published by the AIP Publishing

\section{Over 700 papers \& presentations on} multiphysics simulation 


\title{
Multimodal system for harvesting magnetic and mechanical energy
}

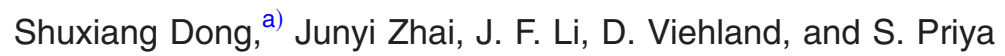 \\ Department of Materials Science and Engineering, Virginia Tech, Blacksburg, Virginia 24061, USA
}

(Received 21 July 2008; accepted 23 August 2008; published online 11 September 2008)

\begin{abstract}
In this letter, we investigate a multimodal system for simultaneous energy harvesting from stray magnetic and mechanical energies by combining magnetoelectric and piezoelectric effects. The system consists of a cantilever beam with tip mass and a magnetoelectric laminate attached in the center of the beam. At 2 Oe magnetic field and mechanical vibration amplitude of $50 \mathrm{mg}$, both at frequency of $20 \mathrm{~Hz}$, the system was found to generate open circuit output voltage of $8 \mathrm{~V}_{\text {P.P. }}$. An equivalent circuit model is proposed that predicts a summation effect for both mechanical and magnetic energies. (C) 2008 American Institute of Physics. [DOI: 10.1063/1.2982099]
\end{abstract}

There is an increasing demand for energy harvesting (EH) systems in mobile electronics and sensor networks. EH systems are required not only to serve as supplemental power source but also in some cases even to replace the batteries such as in implantable networks. Similarly, periodic replacement of batteries in distributed sensor networks is expensive and a tedious operation and would benefit from the development of EH based recharging mechanism. ${ }^{1,2}$ This can happen in various scenarios such as industrial machines, but our emphasis is on undersea power sources. In the future, these systems will be deployable in undersea conditions to simultaneously capture earth's magnetic field and wave energy. In the absence of waves, unmanned undersea vehicles and buoys deployed in deep water could also act as ac magnetic field source. The dc field can also be superimposed by inbuilt magnetic foils which can easily provide $\sim 5$ Oe to overcome the limitation of earth's magnetic field. Furthering this objective in this letter, we report an EH device fabricated from high-permeability magnetostrictive $\mathrm{FeBSiC}$ alloy ribbons laminated with piezoelectric $\mathrm{Pb}(\mathrm{Zr}, \mathrm{Ti}) \mathrm{O}_{3} \quad(\mathrm{PZT})$ fibers. ${ }^{3}$ Our results clearly demonstrate that this laminate composite can synchronously harvest both stray mechanical and magnetic energies. This is a significant advancement toward improving the energy density of current EH systems.

The schematic design of the fabricated energy harvester is illustrated in Fig. 1(a) and a picture of prototype is shown in Fig. 1(b). In this magnetoelectric (ME) laminate configuration, two PZT fiber layers with push-pull-type symmetric polarization units ${ }^{3}$ were laminated together with four magnetostrictive $\mathrm{FeBSiC}$ ribbons. This design allows to utilize mechanisms simultaneously: (i) magnetoelastoelectric effect, ${ }^{4-7}$ where a stray magnetic field $H$ can excite longitudinal strain through magnetostrictive effect of $\mathrm{FeBSiC}$, and (ii) piezoelectric effect, where mechanical vibrations can create strain. The two responses can be combined together to convert composite strain into electricity. Since the conversion occurs through elastic interactions, it is possible to realize additive effect.

Assuming that an external ac magnetic field $H_{\mathrm{ac}}$ and an external mechanical force $F$ of frequency $\omega$ are synchronously applied to the laminate, longitudinal and/or bending

\footnotetext{
a) Also at Advanced Materials and Nanotechnology, College of Engineering, Peking University, Beijing 100871, China. Electronic mail: sxdong@ coe.pku.edu.cn.
}

vibration modes will be excited. We can model the energy harvesting capabilities from excitation of these modes by using an equivalent approach, ${ }^{8}$ as shown in Fig. 1(c). Correspondingly, the induced voltage $\left(V_{\text {induced }}\right)$ across the dielectric layer under open circuit condition can be given as

$$
V_{\text {induced }}=-\phi_{p}\left(\frac{Z_{c}}{Z_{m}}\right)\left(F+\phi_{m} H\right)
$$

where $\varphi_{p}$ is the electromechanical coupling factor, $\varphi_{m}$ the magnetoelastic coupling factor, $Z_{C}$ the capacitance impedance $\left(Z_{c}=1 / j \omega p C_{0}\right)$, and $Z_{m}$ the mechanical impedance. ${ }^{8}$ The negative "-" indicates the reverse phase between the applied $F$ (or $H$ ) and the induced voltage $V_{\text {induced }}$. The induced voltage in Eq. (1) can clearly be seen to be a sum effect from two contributions: (i) a $F$-induced voltage, via a mechanical-to-electric conversion $\varphi_{p}$, which is the first contribution in Eq. (1) and (ii) a $H$-induced voltage, via a magnetoelastoelectric conversion $\varphi_{m} \varphi_{p}$, which is the second contribution in Eq. (1). Please note that $H$ - or $F$-induced
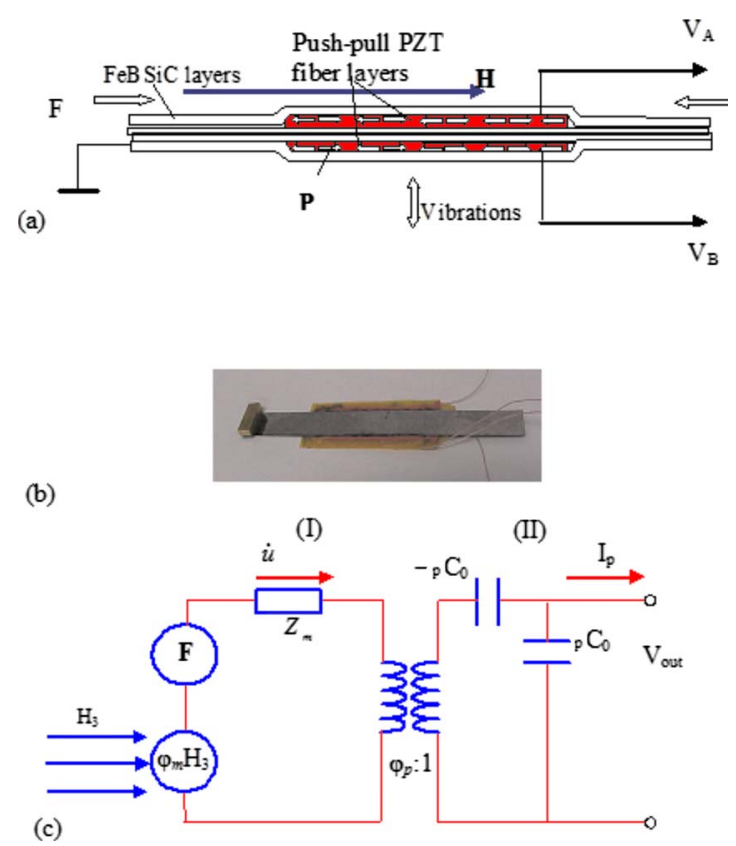

FIG. 1. (Color online) Conceptual illustration of the ME energy harvester: (a) schematic of the ME laminate configuration with p polarization units, (b) photo of the ME laminate prototype, and (c) equivalent circuit model for a bimechanism of EH. ${ }^{8}$ 


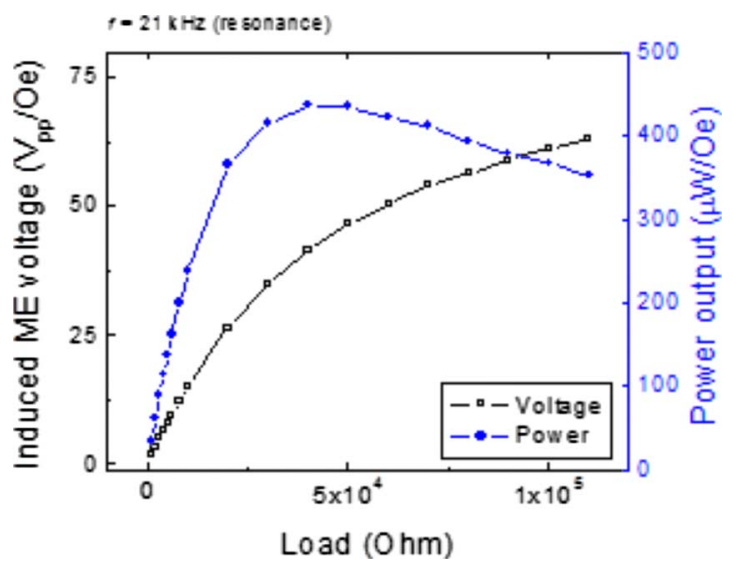

FIG. 2. (Color online) Voltage induced by "stray" magnetic field across our prototype as a function of electrical load.

voltages are related to the ratio of the capacitive impedance of the piezoelectric layer to the mechanical impedance $Z_{m}$ of the entire ME laminate. The mechanical impedance of the laminate should be adjusted in order for effective transfer of stress to match that of the vibration source. Thus, the variables for obtaining large induced voltages are high coupling factors, low damped capacitance which refers to higher piezoelectric voltage coefficient, and higher ME coupling.

The ME laminates were mechanically excited by a vibration testing system that consists of shaker, lock-in amplifier, power amplifier, function generator, and data acqusition interface with a personal computer (PC). A dSPACE shaker was powered by using the Hewlett-Packard bipolar power amplifier $6826 \mathrm{~A}$ and function generator. The generated mechanical excitation was monitored by accelerometer (PCB Piezotronics Model\# U352C22/meter 482A16). Alternating magnetic field $H_{\text {ac }}$ was generated by using Helmholtz coils powered directly by function generator. The voltage generated from the laminate composite was measured by using a digital oscilloscope across a known external load. Figure 2 shows the $H$-induced voltage and the corresponding power as a function of load $\left(P=V_{\mathrm{rms}}^{2} / \mathrm{R}\right)$. The data were measured at the first longitudinal resonance frequency of $\sim 21 \mathrm{kHz}$ without any mechanical vibration. Under an ac magnetic field of

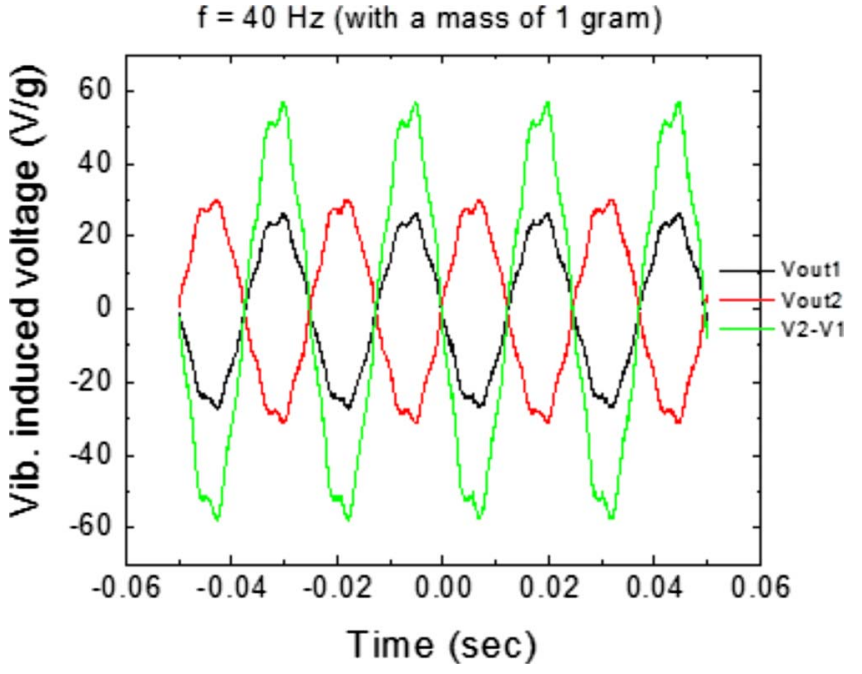

FIG. 3. (Color online) Voltage induced by stray vibration across our prototype as a function of time.

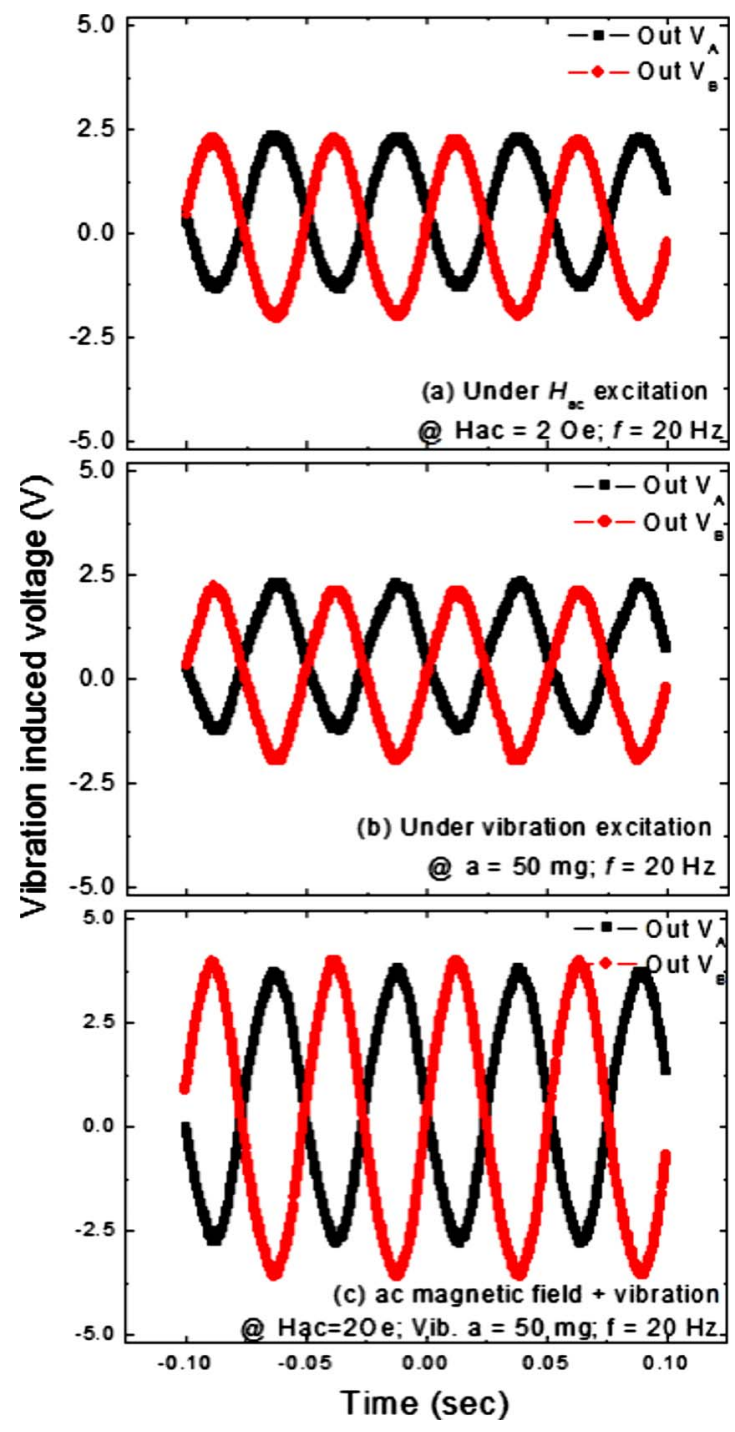

FIG. 4. (Color online) Experimental confirmation of the bimechanism for magnetic field and mechanical vibration EH: (a) under only a stray magnetic excitation of $H_{a c}=2$ Oe $(f=20 \mathrm{~Hz})$, (b) under only a stray vibrational excitation of $a=50 \mathrm{mg}(f=20 \mathrm{~Hz})$, and (c) under both stray magnetic and vibrational excitations.

$1 \mathrm{Oe}$, the maximum induced voltage was $\sim 63 \mathrm{~V}_{\text {p.p. }} / \mathrm{Oe}$; correspondingly, the harvested power output was $420 \mu \mathrm{W} / \mathrm{Oe}$ across a $50 \mathrm{k} \Omega$ load. By accounting for the ME laminate's volume $\left(\sim 0.2 \mathrm{~cm}^{3}\right)$, the output power density can be estimated to be $2.1 \mathrm{~mW} / \mathrm{Oe} \mathrm{cm}^{3}$. This power density is quite high, compared to other types of energy harvesters. ${ }^{1,2,9-11}$ However, there are not many sources emitting stray magnetic fields at this high frequency. Thus, we used a cantilever beam structure and varied the tip mass to lower the operating frequency of the system in the range of $20-40 \mathrm{~Hz}$. The size of the beam was $100 \mathrm{~mm}$ in length, $12 \mathrm{~mm}$ in width, and 0.3 $\mathrm{mm}$ in thickness, and selected tip mass was $1 \mathrm{~g}$.

Figure 3 shows the voltage induced by an applied mechanical vibration, via the direct piezoelectric effect. These data were taken at a bending resonance frequency of $40 \mathrm{~Hz}$ under a vibration acceleration of $1 g$. Measurements were made by mounting the laminate shown in Fig. 1 on top of a shaker, where it was in a cantileverlike configuration, with its free end attached to a small mass of $1 \mathrm{~g}$. The two voltage outputs (from the two piezolayers) generated by an acceleration of $1 g$ were $\sim 60 \mathrm{~V}_{\text {p.p. }}$; correspondingly, the calculated 
power density was $\sim 400 \mu \mathrm{W} / \mathrm{g} \mathrm{cm}^{3}$ at an external load of $3 \mathrm{M} \Omega$. The differential voltage output from these two piezoelectric fiber layers could be as high as $120 \mathrm{~V}_{\text {p.p. }}$, as can be seen in Fig. 3. Practically, the output signal from piezoelectric layers can be separately rectified and conditioned before combining together.

The data presented in Figs. 2 and 3 demonstrate that our ME laminates have capability to harvest both magnetic and mechanical energy. Figure 4 shows the combined response of the system. Figure 4(a) shows the voltage induced at the first bending mode by an applied magnetic field of $H_{\mathrm{ac}}$ $=2$ Oe at $f=20 \mathrm{~Hz}$, without any mechanical vibrations. The output voltages $\mathrm{V}_{\text {out1 }}$ and $\mathrm{V}_{\text {out2 }}$ under the open circuit condition were $\sim 4 \mathrm{~V}_{\text {p.p. }}$ (or $\left.\sim 2 \mathrm{~V}_{\text {p.p. }} / \mathrm{Oe}\right)$. Correspondingly, the magnetically generated electric power at $f=20 \mathrm{~Hz}$ and $R=5 \mathrm{M} \Omega \mathrm{m}$ is $\sim 0.5 \mu \mathrm{W} / \mathrm{Oe} \mathrm{cm}^{3}$. Figure $4(\mathrm{~b})$ shows the voltage induced at the first bending mode by a mechanical vibration with an acceleration of $50 \mathrm{mg}\left(10^{-3} \times 9.8 \mathrm{~m} / \mathrm{s}^{2}\right)$ at $f=20 \mathrm{~Hz}$ without any applied magnetic field. The output voltages $\mathrm{V}_{\text {out1 }}$ and $\mathrm{V}_{\text {out2 }}$ under the open circuit condition were found to be of the order of $\sim 4 \mathrm{~V}_{\text {p.p. }}$ ( or $\sim 80 \mathrm{~V}_{\text {p.p. }} / \mathrm{g}$ ). Correspondingly, the mechanically generated electric power at $f=20 \mathrm{~Hz}$ and $R=5 \mathrm{M} \Omega \mathrm{m}$ is also around $0.5 \mu \mathrm{W} / \mathrm{Oe} \mathrm{cm}^{3}$. Figure 4(c) shows the voltage induced under a simultaneous magnetic field of $H_{\mathrm{ac}}=2$ Oe and a mechanical vibration of amplitude $50 \mathrm{mg}$. This figure shows that the output voltages were doubled relative to a single source to $8 \mathrm{~V}_{\text {p.p. }}$. The data clearly illustrate that the laminates respond to both the external magnetic field and mechanical vibrations. In our case, both the external inputs were imposed at same frequency, which enhanced the bending stress on the composites resulting in doubling of the generated electric charge.
In summary, we report a multimodal system that can be used for harvesting mechanical vibration and magnetic energies simultaneously. We show that the generated electric energy is sum effect from magnetoelastoelectric and piezoelectric contributions. This design has significant promise in designing EH components for the undersea conditions where there is always presence of ocean waves and stray magnetic field. The proposed prototype energy harvester can be further improved by incorporating the self-tuning capability. In narrow bandwidth vibration condition, this could be accomplished by designing the mechanical filter directly into the transducer mounting assembly.

The authors gratefully acknowledge the support from ONR.

${ }^{1}$ P. Glynne-Jones and N. M. White, Sens. Rev. 21, 91 (2001).

${ }^{2}$ H. A. Sodano, D. J. Inman, and G. Park, Shock and Vibration Digest 36, 197 (2004).

${ }^{3}$ S. X. Dong, J. Zhai, J. Li, and D. Viehland, Appl. Phys. Lett. 89, 252904 (2006).

${ }^{4}$ C. W. Nan, M. I. Bichurin, S. X. Dong, D. Viehland, and G. Srinivasan, J. Appl. Phys. 103, 031101 (2008).

${ }^{5}$ R. Ramesh and N. A. Spaldin, Nat. Mater. 6, 21 (2007).

${ }^{6}$ W. Eerenstein, N. D. Mathur, and J. F. Scott, Nature (London) 442, 759 (2006).

${ }^{7}$ M. Fiebig, J. Phys. D 38, R123 (2005).

${ }^{8}$ S. X. Dong, J. F. Li, and D. Viehland, IEEE Trans. Ultrason. Ferroelectr. Freq. Control 50, 1253 (2003).

${ }^{9}$ S. P. Beeby, M. J. Tudor, and N. M. White, Meas. Sci. Technol. 17, R175 (2006).

${ }^{10} \mathrm{~L}$. Wang and F. G. Yuan, Proceedings of the 14th International Symposium on SPIE Smart Structures and Materials \& NDE and Health Monitoring, 2007 (unpublished), p. SSN07.

${ }^{11}$ S. Priya, J. Electroceram. 19, 165 (2007). 